\title{
Genetic diversity of morphological responses and the relationships among Asia aromatic rice (Oryza sativa L.) cultivars
}

\author{
Nguyen Loc Hien ${ }^{1}$, Wakil Ahmad Sarhadi ${ }^{1}$, Yosei Oikawa ${ }^{2}$ and Yutaka Hirata ${ }^{1, *}$ \\ Laboratory of Plant Genetics and Biotechnology, United Graduate School of Agriculture Science, Tokyo University of Agriculture and \\ Technology (TUAT), 3-5-8 Saiwai, Fuchu, Tokyo 183-8509, Japan. \\ ${ }^{2}$ Department of International Environmental and Agricultural Science, TUAT, Tokyo 183-8509, Japan. \\ *Corresponding author; Tel/Fax: +81-42-367-5625, E-mail: yhirata@cc.tuat.ac.jp.
}

\begin{abstract}
An investigation was conducted to determine the extent of diversity and relationships among 36 aromatic rice cultivars collected from Asia. Characterization for 22 morphological characters with 101 morphometric descriptors was carried out. High and comparative levels of phenotypic variation using phenotypic frequency distribution and Shannon-Weaver diversity index were found between countries of origin. Most traits were polymorphic except to ligule color. Grain size, grain shape, culm strength, plant height and secondary branching contributed the highest mean diversity indices $\left(H^{\prime}=0.91,0.88\right.$, $0.87,0.82$ and 0.83 , respectively). For trait groups, highest diversity was found in grain and culm traits $\left(H^{\prime}=1.00\right.$ and 0.96 , respectively). Populations from Vietnam were more diverse than others $\left(H^{\prime}=0.92\right)$ whereas populations from India and Thailand displayed lower diversity indices $\left(\mathrm{H}^{\prime}=0.46\right.$ and 0.49 , respectively). No clear association was detected between phenotypic diversity and countries of origin. Five clusters of 36 genotypes based on Euclidean distance were observed with 1 to 22 cultivars per group. The results of the character distribution and phenotypic diversity analysis permitted some broad generalization about collection and conservation of aromatic rice cultivars. This also indicated the presence of important genes for future aromatic rice breeding purposes. Despite the limitation in estimating total genetic variation, the present study indicated that morphological traits were useful for preliminary evaluation and can be used as a general approach for assessing genetic diversity among morphologically distinguishable aromatic rice cultivars.
\end{abstract}

Key words: Oryza sativa L., aromatic rice, diversity index, morphological trait, phenotypic frequency

\section{INTRODUCTION}

The high quality rice (Oryza sativa L.) production has been significantly increased in recent years. Aromatic rice cultivars are rated best in quality and fetch much higher price than non-aromatic rice cultivars. At present, the consumption from the Middle East, Europe, Australia and the United States of America in displaying a preference for aromatic rice grown in Asian has been increasing. Given this demand for aromatic rice, rice breeders have an interest in understanding the genetic resources of aromatic rice cultivars. Most of aromatic rice cultivars are traditional rice varieties which have tall stature, low yield, photoperiod-sensitivity, susceptible to disease and pest and unresponsive to fertilizer. However, due to the favourite flavour and some other dominant grain quality characteristics, this is the important resource for breeding and improving the aromatic rice cultivars for diverse demands of consumers in the world. Selection of the right type of cultivar is most important factor for optimizing aromatic rice production.

In order to succeed a plant breeding program and to face up to unforeseen breeding challenges in the changing environment, a wider genetic base of germplasm is a prerequisite. Phenotypic and genetic variation within and between countries and regions have examined for several important crop species such as barley (Ruiz et al. 1997) and soybean (Perry and McIntosh, 1991). Therefore, estimates of genetic diversity and the relationships among cultivars are very useful for facilitating the efficient germplasm collection and management. Many tools are now available for studying the variability and relationships among cultivars including isozymes and storage protein analysis and molecular markers linked to particular traits. Morphological traits have a number of limitations including low polymorphism, low heritability, late 
expression and vulnerability to environmental influences (Smith and Smith, 1989). However, morphological characterization is the first step in the classification and evaluation of the germplasm (Smith and Smith, 1989; Smith et al. 1991). Qualitative characters are important for plant description (Kurlovich, 1998) and are influenced by consumer preference, socio-economic scenario and natural selection. Several morphological characters are the major determining factors of rice grain yield. The recognition of genetic variability in any character involved in the yield synthesis provides scope to the possibility of improvement and breeding of aromatic rice. The genetic differentiation among the populations is also useful for breeding new aromatic rice cultivars possessing desire traits.

Up to now, no more systematic study has been carried out to survey the extent of genetic variation and the relationships between various agro-ecotypes of aromatic rice. It is necessary to assess the patterns of character variation and distribution of aromatic rice in different agro-ecological areas. The main objective of this study was to characterize and classify the diversity for twenty-two qualitative traits in aromatic rice cultivars collected from six South and Southeast Asia countries.

\section{MATERIALS AND METHODS}

\section{Plants materials and cultivation practices}

Thirty-six Indica aromatic rice cultivars collected from six Asian countries including Afghanistan, Cambodia, India, Myanmar, Thailand and Vietnam were used. Some studied varietal characters are shown in Table 1. Confirmation test for aromatic character after harvesting was done by chewing individual grains and/or smelling cooked rice with or without enhancement of $1.7 \% \mathrm{KOH}$ solution (Sood and Siddiq, 1978).

Total of 36 cultivars were grown under uniform conditions in Honmachi experimental paddy field and greenhouse at Tokyo University of Agriculture and Technology (Japan). Rice seeds after soaking in warm water at $52^{\circ} \mathrm{C}$ and incubating overnight were sown in plastic tray. Seedlings at 17 days after sowing were transplanted at space of $20 \times 20 \mathrm{~cm}$. A randomized complete block design with two replications was used. The fertilizer dosage was $70 \mathrm{~N}-40 \mathrm{P}-70 \mathrm{~K} \mathrm{~kg} \mathrm{ha}^{-1}$ during the growing season each year. The fertilizer was applied for the basal dressing and top dressing. Water management and pest control were paid attention everyday. Grain samples of photoperiod sensitive cultivars (detailed in Table 1) were harvested from greenhouse.

\section{Morphological characterization}

A total of 22 morphological traits listed in Table 2 (24 including width of grain and growth duration) were evaluated following descriptors for rice (IRRI-IBPGR, 1980). The traits were grouped into five categories: (1) Leaf characteristics below the flag leaf, (2) Ligule characters, (3) Culm characters, (4) Panicle characters and (5) Grain characters. The evaluation methods for the respective traits were briefly described in Table 2 . All cultivars were characterized for 101 morphometric descriptors of 22 morphological traits from early vegetative stage up to the harvest stage, and then were transformed to binary data in which the presence of a descriptor was scored as ' 1 ' and its absence as ' 0 ' for each of morphology characteristics. Characterization was recorded on 10 plants per cultivar per replication (consist of 720 plants for 36 cultivars) except for plant height, sterile lemma length, grain length and width using 20 plants per cultivar per replication (consist of 1440 plants for 36 cultivars).

\section{Data analysis}

The proportions of the different classes for each trait and countries of origin were calculated. Origin of cultivars (country) was used as classifying variables. The phenotypic frequency data of the 22 traits calculated by the Shannon-Weaver diversity index H (Hutchenson, 1970), is defined as:

$$
\mathrm{H}=-\sum_{\mathrm{i}=1}^{\mathrm{n}} \mathrm{P}_{\mathrm{i}} \ln \mathrm{P}_{\mathrm{i}},
$$

where $\mathrm{n}$ is the number of phenotypic classes for a character and $\mathrm{P}_{\mathrm{i}}$ is the relative frequency in the $\mathrm{i}^{\text {th }}$ class of the $\mathrm{j}^{\text {th }}$ trait. $\mathrm{H}$ was estimated for each trait, group of traits and country group. In order to keep the values of $\mathrm{H}$ in the range of $0-1$, each value of $\mathrm{H}$ was divided by its maximum value $\ln (\mathrm{n})$.

The average diversity $\mathrm{H}^{\prime}$ over $\mathrm{j}$ traits was estimated as:

$$
H^{\prime}=\frac{\sum H_{j}}{j}
$$

A one-way analysis of variance of H' index was carried out for each group of trait using countries as classifying variable. These were treated as fixed effects and populations as random. To group the cultivars based on morphological dissimilarity, cluster analysis was conducted on the Euclidean distance matrix with the unweighted pair group average method (UPGMA) using software package STATISTICA version 5.5. 
Table 1. List of 36 Indica aromatic rice cultivars.

\begin{tabular}{|c|c|c|c|c|c|}
\hline Code & Cultivars & Origin & Ecology $^{b}$ & Growth duration (days) & Other \\
\hline A-1 & Bala Baghlan & Afghanistan & IRR & 130 & \\
\hline $\mathrm{A}-2$ & Bala Doshi & Afghanistan & IRR & 128 & \\
\hline A-3 & Luke Andrab & Afghanistan & IRR & 75 & \\
\hline $\mathrm{A}-4$ & Monda Laghman & Afghanistan & IRR & 120 & \\
\hline$A-5$ & Pashadi Laghman & Afghanistan & IRR & 125 & \\
\hline $\mathrm{C}-1$ & Phkarum Doul & Cambodia & RFL & $120-160$ & Photoperiod sensitive \\
\hline $\mathrm{C}-2$ & Phkarum Check & Cambodia & RFL & $120-160$ & Photoperiod sensitive \\
\hline $\mathrm{C}-3$ & Phkarum Chang & Cambodia & RFL & $120-160$ & Photoperiod sensitive \\
\hline $\mathrm{C}-4$ & Senpidao & Cambodia & IRR & 135 & \\
\hline $\mathrm{I}-1$ & Basmati $370-1^{\text {a }}$ & India & IRR & 160 & \\
\hline $\mathrm{I}-2$ & Basmati $370-2^{a}$ & India & IRR & 158 & \\
\hline $\mathrm{I}-3$ & Surjamkhi & India & IRR & 110 & \\
\hline M-1 & Nga Kywe Taung Pyan & Myanmar & IRR/RFL & 155 & Photoperiod sensitive \\
\hline M-2 & Nga Kywe Yin & Myanmar & IRR/RFL & 135 & Photoperiod sensitive \\
\hline M-3 & Mee Done Hmwe & Myanmar & IRR/RFL & 145 & Photoperiod sensitive \\
\hline M-4 & Mee Done Taung & Myanmar & IRR/RFL & 135 & Photoperiod sensitive \\
\hline M-5 & Paw San Shwe War & Myanmar & IRR/RFL & 130 & Photoperiod sensitive \\
\hline $\mathrm{T}-1$ & Daw Dam & Thailand & UPL & 115 & \\
\hline $\mathrm{T}-2$ & Jasmine 85 & Thailand & IRR & 115 & \\
\hline $\mathrm{T}-3$ & Khao Dawk Mali 105 & Thailand & UPL & $120-160$ & Photoperiod sensitive \\
\hline $\mathrm{V}-1$ & Hoa Lai & Vietnam & IRR/RFL & $120-160$ & Photoperiod sensitive \\
\hline $\mathrm{V}-2$ & Me Huong 2 & Vietnam & IRR/RFL & $120-160$ & Photoperiod sensitive \\
\hline V-3 & MTL250 $-1^{\text {a }}$ & Vietnam & IRR & 135 & \\
\hline $\mathrm{V}-4$ & MTL250 $-2^{a}$ & Vietnam & IRR & 135 & \\
\hline $\mathrm{V}-5$ & Nang Thom Cho Dao $1^{a}$ & Vietnam & IRR/RFL & $120-160$ & Photoperiod sensitive \\
\hline $\mathrm{V}-6$ & Nang Thom Cho Dao $2^{\mathrm{a}}$ & Vietnam & IRR/RFL & $120-160$ & Photoperiod sensitive \\
\hline $\mathrm{V}-7$ & Nang Thom Cho Dao $3^{a}$ & Vietnam & IRR/RFL & $120-160$ & Photoperiod sensitive \\
\hline $\mathrm{V}-8$ & Nang Thom Cho Dao $4^{a}$ & Vietnam & IRR/RFL & $120-160$ & Photoperiod sensitive \\
\hline V-9 & Nang Thom Duc & Vietnam & IRR/RFL & $120-160$ & Photoperiod sensitive \\
\hline V-10 & Nang Thom Som & Vietnam & IRR/RFL & $120-160$ & Photoperiod sensitive \\
\hline V-11 & Tau Huong 2 & Vietnam & IRR/RFL & $120-160$ & Photoperiod sensitive \\
\hline $\mathrm{V}-12$ & Thanh Tra & Vietnam & $\mathrm{IRR} / \mathrm{RFL}$ & $120-160$ & Photoperiod sensitive \\
\hline V-13 & Gie Vang 2 & Vietnam & IRR/RFL & $120-160$ & Photoperiod sensitive \\
\hline V-14 & Nang Huong 2 & Vietnam & IRR/RFL & $120-160$ & Photoperiod sensitive \\
\hline V-15 & Nang Tet 2 & Vietnam & IRR/RFL & $120-160$ & Photoperiod sensitive \\
\hline $\mathrm{V}-16$ & VD20 & Vietnam & IRR & 134 & \\
\hline
\end{tabular}

${ }^{a}$ Different samples obtained from one original cultivar through estimating differences of morphologic and agronomic characteristics and aromatic strength. IRR = Irrigated; RFL = Rainfed Lowland; UPL = Upland 
Table 2. Descriptors and their codes of 22 morphological traits examined.

\begin{tabular}{|c|c|c|}
\hline Trait & Description and code $^{a}$ & Growth stage \\
\hline \multicolumn{3}{|l|}{ Leaf (below the flag leaf) } \\
\hline Blade pubescence & Glabrous (1), Intermediate (2), Pubescent (3) & Late vegetative stage \\
\hline Basal leaf sheath color & Green (1), Purple lines (2), Light purple (3), Purple (4) & Early to late vegetative stage \\
\hline Flag leaf angle & Erect (1), Intermediate (3), Horizontal (5), Descending (7) & After heading \\
\hline \multicolumn{3}{|l|}{ Ligule } \\
\hline Color & White (1), Purple lines (2), Purple (3) & Late vegetative stage \\
\hline Shape & Acute to acuminate (1), 2-cleft (2), Truncate (3) & Late vegetative stage \\
\hline Collar color & Pale green (1), Green (2), Purple (3) & Late vegetative stage \\
\hline Auricle color & Pale green (1), Purple (2) & Late vegetative stage \\
\hline \multicolumn{3}{|l|}{ Culm } \\
\hline Plant height & $\begin{array}{l}\text { No higher than } 110 \mathrm{~cm}(1), \text { From } 110 \mathrm{~cm} \text { to } 130 \mathrm{~cm}(5), \text { Higher } \\
\text { than } 130 \mathrm{~cm}(9)\end{array}$ & Maturity stage \\
\hline Angle & $\begin{array}{l}\text { Erect (1),Intermediate (3), Open (5), Spreading (7), Procumbent } \\
\text { (9) }\end{array}$ & Flowering to maturity stage \\
\hline Strength (Lodging resistance) & $\begin{array}{l}\text { Strong (1), Moderately strong (3), Intermediately (5), Weak (7) } \\
\text { Very weak (9) }\end{array}$ & Flowering to maturity stage \\
\hline \multicolumn{3}{|l|}{ Panicle } \\
\hline Type & Compact (1), Intermediate (5), Open (9) & Near maturity to maturity stage \\
\hline Secondary branching & Absent (0), Light (1), Heavy (2), Clustering (3) & Maturity stage \\
\hline \multicolumn{3}{|l|}{ Grain } \\
\hline Awning & $\begin{array}{l}\text { Absent (0), Short and partly (1), Short and fully (5), Long and } \\
\text { partly (7), Long and fully (9) }\end{array}$ & Maturity stage \\
\hline Awn color & $\begin{array}{l}\text { No awn (0), Straw (1), Gold (2), Brown (Tawny) (3), Red (4), } \\
\text { Purple (5), Black (6) }\end{array}$ & Maturity stage \\
\hline Apiculus color & $\begin{array}{l}\text { White (1), Straw (2), Brown (Tawny) (3), Red (4), Red apex (5), } \\
\text { Purple (6), Purple apex (7) }\end{array}$ & Maturity stage \\
\hline Lemma and palea color & $\begin{array}{l}\text { Straw (0), Gold and/or gold furrows on straw (1), Brown spots } \\
\text { on brown (2), Brown furrows on straw (3), Brown (Tawny) (4), } \\
\text { Reddish to light purple (5), Purple spots on straw (6), Purple } \\
\text { furrows on straw (7), Purple (8), Black (9), White (10) }\end{array}$ & Maturity stage \\
\hline Lemma and palea pubescence & $\begin{array}{l}\text { Glabrous (1), Hairs on lemma keel (2), Hairs on upper portion (3), } \\
\text { Short hairs (4), Long hairs (5) }\end{array}$ & Maturity stage \\
\hline Sterile lemma color & Straw (Yellow) (1), Gold (2), Red (3), Purple (4) & Maturity stage \\
\hline Sterile lemma length & $\begin{array}{l}\text { Short (no longer than } 1.5 \mathrm{~mm})(1), \text { Medium }(1.6-2.5 \mathrm{~mm})(3) \text {, } \\
\text { Long (longer than } 2.5 \mathrm{~mm} \text { but shorter than the lemma) (5), Extra } \\
\text { long (Equal to or longer than the lemma) (7), Asymmetrical (9) }\end{array}$ & Maturity stage \\
\hline Grain size (Grain length (L)) & $\begin{array}{l}\text { Extra long } \mathrm{L}>7.5 \mathrm{~mm}(1) \text {, Long } 6.61<\mathrm{L}<7.50 \text { (2), Medium } 5.51 \\
<\mathrm{L}<6.60 \text { (3), Short } \mathrm{L}<5.50 \text { (4) }\end{array}$ & Maturity stage \\
\hline $\begin{array}{l}\text { Grain shape (Grain length }(\mathrm{L}) / \\
\text { Grain width }(\mathrm{W}) \text { ratio) }\end{array}$ & $\begin{array}{l}\text { Slender and long } \mathrm{L} / \mathrm{W}>3.0(1) \text {, Medium } 2.1<\mathrm{L} / \mathrm{W}<3.0(2) \text {, } \\
\text { Bold } 1.1<\mathrm{L} / \mathrm{W}<2.0(3) \text {, Round } \mathrm{L} / \mathrm{W}<1.0 \text { (4) }\end{array}$ & Maturity stage \\
\hline Pericarp color & $\begin{array}{l}\text { White (1), Light brown (2), Speckled brown (3), Brown (4), Red } \\
\text { (5), Variable purple (6), Purple (7) }\end{array}$ & Maturity stage \\
\hline
\end{tabular}

${ }^{\mathrm{a}}$ For more detail see IBPGR-IRRI Rice Advisory Committee (1980). 
Table 3. Percentage and no. of plants (in parentheses) in each phenotypic class.

\begin{tabular}{|c|c|c|c|c|c|c|c|c|}
\hline \multirow{2}{*}{ Trait } & \multirow{2}{*}{ Class } & \multicolumn{6}{|c|}{ Country } & \multirow{2}{*}{ Total } \\
\hline & & Afg & Cam & Ind & Mya & Tha & Vie & \\
\hline \multirow[t]{3}{*}{ Leaf blade pubescence } & 1 & $60(60)$ & $25(20)$ & $67(40)$ & $20(20)$ & $100(60)$ & $31(100)$ & $42(300)$ \\
\hline & 2 & $40(40)$ & $75(60)$ & $0(0)$ & $80(80)$ & $0(0)$ & $69(220)$ & $55(400)$ \\
\hline & 3 & $0(0)$ & $0(0)$ & $33(20)$ & $0(0)$ & $0(0)$ & $0(0)$ & $3(20)$ \\
\hline \multirow[t]{4}{*}{ Basal leaf sheath color } & 1 & $80(80)$ & $100(80)$ & $67(40)$ & $100(100)$ & $100(60)$ & $94(300)$ & $92(660)$ \\
\hline & 2 & $0(0)$ & $0(0)$ & $0(0)$ & $0(0)$ & $0(0)$ & $0(0)$ & $0(0)$ \\
\hline & 3 & $0(0)$ & $0(0)$ & $0(0)$ & $0(0)$ & $0(0)$ & $0(0)$ & $0(0)$ \\
\hline & 4 & $20(20)$ & $0(0)$ & $33(20)$ & $0(0)$ & $0(0)$ & $6(20)$ & $8(60)$ \\
\hline \multirow[t]{4}{*}{ Flag leaf angle } & 1 & $20(20)$ & $25(20)$ & $0(0)$ & $0(0)$ & $0(0)$ & $19(60)$ & $14(100)$ \\
\hline & 3 & $60(60)$ & $75(60)$ & $67(40)$ & $100(100)$ & $67(40)$ & $81(260)$ & $78(560)$ \\
\hline & 5 & $20(20)$ & $0(0)$ & 33 (20) & $0(0)$ & 33 (20) & $0(0)$ & $8(60)$ \\
\hline & 7 & $0(0)$ & $0(0)$ & $0(0)$ & $0(0)$ & $0(0)$ & $0(0)$ & $0(0)$ \\
\hline \multirow[t]{3}{*}{ Ligule color } & 1 & $100(100)$ & $100(80)$ & $100(60)$ & $100(100)$ & $100(60)$ & $100(320)$ & $100(720)$ \\
\hline & 2 & $0(0)$ & $0(0)$ & $0(0)$ & $0(0)$ & $0(0)$ & $0(0)$ & $0(0)$ \\
\hline & 3 & $0(0)$ & $0(0)$ & $0(0)$ & $0(0)$ & $0(0)$ & $0(0)$ & $0(0)$ \\
\hline \multirow[t]{3}{*}{ Ligule shape } & 1 & $20(20)$ & $0(0)$ & $0(0)$ & $0(0)$ & $0(0)$ & $0(0)$ & $3(20)$ \\
\hline & 2 & $80(80)$ & $100(80)$ & $100(60)$ & $100(100)$ & $100(60)$ & $100(320)$ & $97(700)$ \\
\hline & 3 & $0(0)$ & $0(0)$ & $0(0)$ & $0(0)$ & $0(0)$ & $0(0)$ & $0(0)$ \\
\hline \multirow[t]{3}{*}{ Collar color } & 1 & $40(40)$ & $50(40)$ & $67(40)$ & $100(100)$ & $100(60)$ & $63(200)$ & $67(480)$ \\
\hline & 2 & $40(40)$ & $50(40)$ & 33 (20) & $0(0)$ & $0(0)$ & $25(80)$ & 25 (180) \\
\hline & 3 & $20(20)$ & $0(0)$ & $0(0)$ & $0(0)$ & $0(0)$ & $12(40)$ & $8(60)$ \\
\hline \multirow[t]{2}{*}{ Auricle color } & 1 & $80(80)$ & $100(80)$ & $100(60)$ & $100(100)$ & $100(60)$ & 87 (280) & $92(660)$ \\
\hline & 2 & $20(20)$ & $0(0)$ & $0(0)$ & $0(0)$ & $0(0)$ & $13(40)$ & $8(60)$ \\
\hline \multirow[t]{3}{*}{ Plant height } & 1 & $40(80)$ & $25(40)$ & $0(0)$ & $20(40)$ & $33(40)$ & $0(0)$ & $14(200)$ \\
\hline & 2 & $0(0)$ & $0(0)$ & $0(0)$ & $0(0)$ & $34(40)$ & 19 (120) & $11(160)$ \\
\hline & 3 & 60 (120) & 75 (120) & $100(120)$ & 80 (160) & 33 (40) & $81(520)$ & 75 (1080) \\
\hline \multirow[t]{5}{*}{ Culm angle } & 1 & $20(20)$ & $25(20)$ & $0(0)$ & $0(0)$ & $100(60)$ & $100(320)$ & $58(420)$ \\
\hline & 3 & $40(40)$ & $75(60)$ & $100(60)$ & $100(100)$ & $0(0)$ & $0(0)$ & $36(260)$ \\
\hline & 5 & $40(40)$ & $0(0)$ & $0(0)$ & $0(0)$ & $0(0)$ & $0(0)$ & $6(40)$ \\
\hline & 7 & $0(0)$ & $0(0)$ & $0(0)$ & $0(0)$ & $0(0)$ & $0(0)$ & $0(0)$ \\
\hline & 9 & $0(0)$ & $0(0)$ & $0(0)$ & $0(0)$ & $0(0)$ & $0(0)$ & $0(0)$ \\
\hline \multirow[t]{5}{*}{ Culm strength } & 1 & $0(0)$ & $0(0)$ & $0(0)$ & $0(0)$ & $0(0)$ & $19(60)$ & $8(60)$ \\
\hline & 3 & $20(20)$ & $25(20)$ & $33(20)$ & $60(60)$ & $67(40)$ & 38 (120) & 39 (280) \\
\hline & 5 & $0(0)$ & $75(60)$ & $67(40)$ & $20(20)$ & $33(20)$ & $42(140)$ & 39 (280) \\
\hline & 7 & $0(0)$ & $0(0)$ & $0(0)$ & $20(20)$ & $0(0)$ & $0(0)$ & $3(20)$ \\
\hline & 9 & $80(80)$ & $0(0)$ & $0(0)$ & $0(0)$ & $0(0)$ & $0(0)$ & $11(80)$ \\
\hline \multirow[t]{3}{*}{ Panicle type } & 1 & $20(20)$ & $25(20)$ & $0(0)$ & $20(20)$ & $33(20)$ & $0(0)$ & $11(80)$ \\
\hline & 5 & $60(60)$ & $75(60)$ & $33(20)$ & $80(80)$ & $67(40)$ & $100(320)$ & $81(580)$ \\
\hline & 9 & $20(20)$ & $0(0)$ & $67(40)$ & $0(0)$ & $0(0)$ & $0(0)$ & $8(60)$ \\
\hline \multirow[t]{4}{*}{ Secondary branching } & 0 & $0(0)$ & $0(0)$ & $0(0)$ & $0(0)$ & $0(0)$ & $0(0)$ & $0(0)$ \\
\hline & 1 & $40(40)$ & $25(20)$ & $33(20)$ & $40(40)$ & $33(20)$ & $56(180)$ & $44(320)$ \\
\hline & 2 & $40(40)$ & $75(60)$ & $67(40)$ & $60(60)$ & $34(20)$ & $25(80)$ & $42(300)$ \\
\hline & 3 & $20(20)$ & $0(0)$ & $0(0)$ & $0(0)$ & $33(20)$ & $19(60)$ & $14(100)$ \\
\hline \multirow[t]{5}{*}{ Awning } & 0 & $40(40)$ & $100(80)$ & $33(20)$ & $80(80)$ & $100(60)$ & $81(260)$ & $75(540)$ \\
\hline & 1 & $20(20)$ & $0(0)$ & $34(20)$ & $20(20)$ & $0(0)$ & $0(0)$ & $8(60)$ \\
\hline & 5 & $0(0)$ & $0(0)$ & $0(0)$ & $0(0)$ & $0(0)$ & $13(40)$ & $6(40)$ \\
\hline & 7 & $40(40)$ & $0(0)$ & $0(0)$ & $0(0)$ & $0(0)$ & $6(20)$ & $8(60)$ \\
\hline & 9 & $0(0)$ & $0(0)$ & $33(20)$ & $0(0)$ & $0(0)$ & $0(0)$ & $320)$ \\
\hline Awn color & 0 & $40(40)$ & $100(80)$ & $33(20)$ & $80(80)$ & $100(60)$ & $81(260)$ & $75(540)$ \\
\hline & 1 & $0(0)$ & $0(0)$ & $0(0)$ & $0(0)$ & $0(0)$ & $6(20)$ & $3(20)$ \\
\hline & 2 & $0(0)$ & $0(0)$ & $34(20)$ & $0(0)$ & $0(0)$ & $6(20)$ & $640)$ \\
\hline & 3 & $20(20)$ & $0(0)$ & 33 (20) & $20(20)$ & $0(0)$ & $7(20)$ & $11(80)$ \\
\hline & 4 & $0(0)$ & $0(0)$ & $0(0)$ & $0(0)$ & $0(0)$ & $0(0)$ & $0(0)$ \\
\hline & 5 & $40(40)$ & $0(0)$ & $0(0)$ & $0(0)$ & $0(0)$ & $0(0)$ & $5(40)$ \\
\hline & 6 & $0(0)$ & $0(0)$ & $0(0)$ & $0(0)$ & $0(0)$ & $0(0)$ & $0(0)$ \\
\hline
\end{tabular}


Table 3. (continued)

\begin{tabular}{|c|c|c|c|c|c|c|c|c|}
\hline \multirow{2}{*}{ Trait } & \multirow{2}{*}{ Class } & \multicolumn{6}{|c|}{ Country $\left(^{*}\right)$} & \multirow{2}{*}{ Total } \\
\hline & & Afg & Cam & Ind & Mya & Tha & Vie & \\
\hline \multirow[t]{7}{*}{ Apiculus color } & 1 & $20(20)$ & $0(0)$ & $0(0)$ & $0(0)$ & $0(0)$ & $0(0)$ & $3(20)$ \\
\hline & 2 & $20(20)$ & $100(80)$ & $33(20)$ & $40(40)$ & $67(40)$ & $94(300)$ & $69(500)$ \\
\hline & 3 & $20(20)$ & $0(0)$ & $34(20)$ & $60(60)$ & $0(0)$ & $6(10)$ & $17(100)$ \\
\hline & 4 & $0(20)$ & $0(0)$ & $33(20)$ & $0(0)$ & $33(20)$ & $0(0)$ & $6(60)$ \\
\hline & 5 & $0(0)$ & $0(0)$ & $0(0)$ & $0(0)$ & $0(0)$ & $0(0)$ & $0(0)$ \\
\hline & 6 & $40(40)$ & $0(0)$ & $0(0)$ & $0(0)$ & $0(0)$ & $0(0)$ & $5(40)$ \\
\hline & 7 & $0(0)$ & $0(0)$ & $0(0)$ & $0(0)$ & $0(0)$ & $0(0)$ & $0(0)$ \\
\hline \multirow[t]{11}{*}{ Lemma and palea color } & 0 & $40(40)$ & $25(20)$ & $33(20)$ & $0(0)$ & $33(20)$ & $56(180)$ & $39(280)$ \\
\hline & 1 & $40(40)$ & $75(60)$ & $34(20)$ & $40(40)$ & $34(20)$ & $44(140)$ & $44(320)$ \\
\hline & 2 & $0(0)$ & $0(0)$ & $0(0)$ & $20(20)$ & $0(0)$ & $0(0)$ & $3(20)$ \\
\hline & 3 & $20(20)$ & $0(0)$ & $33(0)$ & $20(20)$ & $0(0)$ & $0(0)$ & $8(60)$ \\
\hline & 4 & $0(0)$ & $0(0)$ & $0(0)$ & $20(20)$ & $0(0)$ & $0(0)$ & $3(20)$ \\
\hline & 5 & $0(0)$ & $0(0)$ & $0(0)$ & $0(0)$ & $0(0)$ & $0(0)$ & $0(0)$ \\
\hline & 6 & $0(0)$ & $0(0)$ & $0(0)$ & $0(0)$ & $0(0)$ & $0(0)$ & $0(0)$ \\
\hline & 7 & $0(0)$ & $0(0)$ & $0(0)$ & $0(0)$ & $33(20)$ & $0(0)$ & $3(20)$ \\
\hline & 8 & $0(0)$ & $0(0)$ & $0(0)$ & $0(0)$ & $0(0)$ & $0(0)$ & $0(0)$ \\
\hline & 9 & $0(0)$ & $0(0)$ & $0(0)$ & $0(0)$ & $0(0)$ & $0(0)$ & $0(0)$ \\
\hline & 10 & $0(0)$ & $0(0)$ & $0(0)$ & $0(0)$ & $0(0)$ & $0(0)$ & $0(0)$ \\
\hline \multirow[t]{5}{*}{ Lemma and palea pubescence } & 1 & $20(20)$ & $75(60)$ & $67(40)$ & $80(80)$ & $67(40)$ & 87 (280) & $72(520)$ \\
\hline & 2 & $40(40)$ & $0(0)$ & $33(20)$ & $20(20)$ & $0(0)$ & $0(0)$ & $11(80)$ \\
\hline & 3 & $40(40)$ & $25(20)$ & $0(0)$ & $0(0)$ & $33(20)$ & $13(40)$ & $17(120)$ \\
\hline & 4 & $0(0)$ & $0(0)$ & $0(0)$ & $0(0)$ & $0(0)$ & $0(0)$ & $0(0)$ \\
\hline & 5 & $0(0)$ & $0(0)$ & $0(0)$ & $0(0)$ & $0(0)$ & $0(0)$ & $0(0)$ \\
\hline \multirow[t]{4}{*}{ Sterile lemma color } & 1 & $80(80)$ & $75(60)$ & $33(20)$ & $40(40)$ & 33 (20) & $81(260)$ & $67(480)$ \\
\hline & 2 & $20(20)$ & $25(20)$ & $34(20)$ & $60(60)$ & $34(20)$ & $19(60)$ & 27 (200) \\
\hline & 3 & $0(0)$ & $0(0)$ & $33(20)$ & $0(0)$ & $33(20)$ & $0(0)$ & $6(40)$ \\
\hline & 4 & $0(0)$ & $0(0)$ & $0(0)$ & $0(0)$ & $0(0)$ & $0(0)$ & $0(0)$ \\
\hline \multirow[t]{5}{*}{ Sterile lemma length } & 1 & $20(40)$ & $25(40)$ & $0(0)$ & $20(40)$ & $67(80)$ & $44(280)$ & $33(480)$ \\
\hline & 3 & $40(80)$ & 75 (120) & $33(40)$ & $60(120)$ & 33 (40) & $56(360)$ & $53(760)$ \\
\hline & 5 & $40(80)$ & $0(0)$ & $67(80)$ & $0(0)$ & $0(0)$ & $0(0)$ & $11(160)$ \\
\hline & 7 & $0(0)$ & $0(0)$ & $0(0)$ & $20(40)$ & $0(0)$ & $0(0)$ & $3(40)$ \\
\hline & 9 & $0(0)$ & $0(0)$ & $0(0)$ & $0(0)$ & $0(0)$ & $0(0)$ & $0(0)$ \\
\hline \multirow[t]{4}{*}{ Grain size } & 1 & $60(120)$ & 75 (120) & $67(80)$ & $0(0)$ & $67(80)$ & 19 (120) & $35(520)$ \\
\hline & 2 & $20(40)$ & $25(40)$ & $33(40)$ & $20(40)$ & $33(40)$ & $63(400)$ & $42(600)$ \\
\hline & 3 & $0(0)$ & $0(0)$ & $0(0)$ & $60(120)$ & $0(0)$ & $18(120)$ & $17(240)$ \\
\hline & 4 & $20(40)$ & $0(0)$ & $0(0)$ & $20(40)$ & $0(0)$ & $0(0)$ & $6(80)$ \\
\hline \multirow[t]{4}{*}{ Grain shape } & 1 & $60(120)$ & $100(160)$ & $100(120)$ & $0(0)$ & $67(80)$ & $100(640)$ & $78(1120)$ \\
\hline & 2 & $20(40)$ & $0(0)$ & $0(0)$ & $80(160)$ & 33 (40) & $0(0)$ & $17(240)$ \\
\hline & 3 & $20(40)$ & $0(0)$ & $0(0)$ & $20(40)$ & $0(0)$ & $0(0)$ & $5(80)$ \\
\hline & 4 & $0(0)$ & $0(0)$ & $0(0)$ & $0(0)$ & $0(0)$ & $0(0)$ & $0(0)$ \\
\hline \multirow[t]{7}{*}{ Pericarp color } & 1 & $40(40)$ & $25(20)$ & $33(20)$ & $60(60)$ & $67(40)$ & $100(320)$ & $69(500)$ \\
\hline & 2 & $20(20)$ & $75(60)$ & $67(40)$ & $20(20)$ & 33 (20) & $0(0)$ & $22(160)$ \\
\hline & 3 & $0(0)$ & $0(0)$ & $0(0)$ & $0(0)$ & $0(0)$ & $0(0)$ & $0(0)$ \\
\hline & 4 & $40(40)$ & $0(0)$ & $0(0)$ & $20(20)$ & $0(0)$ & $0(0)$ & $9(60)$ \\
\hline & 5 & $0(0)$ & $0(0)$ & $0(0)$ & $0(0)$ & $0(0)$ & $0(0)$ & $0(0)$ \\
\hline & 6 & $0(0)$ & $0(0)$ & $0(0)$ & $0(0)$ & $0(0)$ & $0(0)$ & $0(0)$ \\
\hline & 7 & $0(0)$ & $0(0)$ & $0(0)$ & $0(0)$ & $0(0)$ & $0(0)$ & $0(0)$ \\
\hline
\end{tabular}

$\left(^{*}\right)$ : Afg: Afghanistan, Cam: Cambodia, Ind: India, Mya: Myanmar, Tha: Thailand, Vie: Vietnam 


\section{RESULTS AND DISCUSSION}

\section{Phenotypic frequencies and traits distribution}

Frequencies of phenotypic classes expressed in percentage for each trait in six countries are shown in Table 3. All considered traits showed markable differences in their distribution and amount of variations within them. Except that ligule color was found to be invariant in all cultivars and populations, the polymorphism was observed and evident in varying degrees at the population level. Many of phenotypic classes were absent in all populations and others dominated in all countries.

\section{Leaf characters}

Individual traits of leaf characters differed in their types of distribution and level of variation. Two classes of glabrous and intermediate leaf blade were presented in similar percentages in the whole collection ( $42 \%$ and $56 \%$, respectively) but with considerable difference in each country. Pubescent type of leaf blade was found in only India populations and displayed rare frequency (2\%) in total of surveyed cultivars. For basal leaf sheath color, only two types of green and purple occurred in this investigation. The highest frequency for green color of basal leaf sheath was $92 \%$. However this category was monomorphic in Cambodia, Myanmar and Thailand populations. Purple color of basal leaf sheath was found in low frequencies in Afghanistan, India and Vietnam cultivars. Flag leaf and flag leaf angle contributes largely to the filling of grains because it supplies photosynthetic products, mainly to the panicle (Yoshida, 1981). Photosynthesis ratio of erect and intermediate flag leaves was higher than horizontal and descending ones. Intermediate angle of flag leaf was dominant trait with $78 \%$ entire collection. Horizontal flag leaf angle occurred in Afghanistan, India and Thailand with $8 \%$ entire collection whereas descending did not present in any populations. Some of Afghanistan, Cambodia and Vietnam cultivars possess the erect flag leaf obtaining to $14 \%$ of estimated cultivars. Particularly those cultivars should be marked for high yielding rice breeding program.

\section{Ligule characters}

Traits of ligule character, in general, tend to distribute in given class of a trait as a predominant phenotypic class. Ligule color was only monomorphism trait in the present study through one hundred percent of estimated samples displayed in white ligule color. For ligule shape, 2-cleft ligule was more frequent than others in the whole collection. Ligule shape of acute to acuminate was found at particularly low frequency in Afghanistan populations. While pale green collar was dominant in entire collection, purple collar found in Afghanistan and Vietnam populations showed at low appearance frequency of comprised $8 \%$. Only pale green auricle color occurred in populations from Cambodia, India, Myanmar and Thailand whereas purple color was observed at Afghanistan and Vietnam populations with frequencies of $20 \%$ and $13 \%$, respectively. Although there is a variation of distribution for ligule characters among populations and countries, adaptive and economic significance in rice production of these traits is not clear known. Genetic diversity of ligule found might be shown as the result of natural selection to these traits.

\section{Culm characters}

The predominant phenotype in all countries was the high plant type. While three classes of plant height (semi-dwarf, intermediate and tall) in Thailand populations represented at similar frequencies (33\%, 34\% and 33\%, respectively), all populations from India have tall plant phenotype. Semidwarf cultivars from Afghanistan, Cambodia, Myanmar and Thailand ( $14 \%$ of whole collection) were useful genetic source for aromatic rice breeding program. Culm angle trait was found more polymorphic in Afghanistan and Cambodia cultivars whereas others possessed only either erect or intermediate types. In this collection, erect culm angle was more dominant (58\%) and there was not occurrence of spreading and procumbent types in any population. Moderately and intermediately culm strength were found popularly in frequency of $39 \%$ and $39 \%$, respectively, in whole collection. Culm strength in Afghanistan cultivars was weaker than others while 80\% populations of Afghanistan cultivars was severe lodging. Some of Myanmar populations were susceptible to lodging in the present collection. Only $8 \%$ cultivars were found to be resistant to lodging and they belonged to Vietnam rice cultivars. Although a high frequency of tall rice cultivars was identified, only $11 \%$ of entire collection lodged. This agrees with the suggestion that short rice plant does not guarantee a high degree of lodging resistance (Ookawa and Ishihara, 1993; Watanabe, 1997; Hien, 2001). Culm strength in rice depends on the physical properties of culm, i.e. stoutness and stiffness of culm, in addition to morphological characters, environmental factors and meteorological conditions (Hien, 2001). Therefore, improvement of aromatic rice cultivars also should focus on both decreasing the plant height and increasing the culm strength.

\section{Panicle characters}

There were three classes of panicle type occurred in all populations, with the intermediate one being the 
predominant type in all countries except for India. A low proportion of open panicle type (8\%) was presented in Afghanistan and India populations. Only Basmati 370 populations from India possessed open panicle type. Panicle type is an important morphological trait which relates partly to yield in rice; however its influence is in association with flag angle trait from heading stage to ripening stage of rice plant. The rice cultivars with compact panicles and erect flag leaf angle are better for both filling grains and tolerance to insects and birds. Few cultivars from Afghanistan and Cambodia possessing both these advantages should be objectives of aromatic rice breeding. Secondary branching of panicle was relatively diverse though the type of no secondary branching was absent in all populations. Light and heavy secondary branching displayed a fairly high proportion (44\% and $42 \%$, respectively) in countries distribution. Only $14 \%$ of the entries had clustering type of panicle.

\section{Grain characters}

The awns in wild rice cultivars are considered to be advantageous because these protect rice grains from animal attack and play an important role in seed dispersal (Takahashi et al. 1986). Except Indian cultivars, awnless phenotype was predominant than others. The highest frequency for awnless grain type was $75 \%$ of total collection. Of $25 \%$ of awned grain also showed a polymorphism of awn color in Afghanistan, India, Myanmar and Vietnam cultivars. Straw apiculus color occurred in $69 \%$ populations was more dominant than others. However this type was abundant in only Cambodia, Thailand and Vietnam aromatic rice. In this group of characters, the popularity of awnless trait may be due to linkage with selective factors for yield and postharvest production. The result suggested the presence of selection advantage to this trait in this collection.

Straw and gold lemma and palea color were frequent compared with others (39\% and 44\%, respectively). Cambodia, Thailand and Vietnam cultivars had only two classes of straw and gold lemma and palea color whereas Myanmar cultivars showed more variation of four classes for lemma and palea color. Glabrous grains were more abundant than the alternate variants (hairy grain) in all populations except to Afghanistan populations. Sterile lemma color and length were diverse in entire collection however, straw sterile lemma with medium length occurred more frequent. Gold color of sterile lemma was dominant in Myanmar cultivars. Sterile lemma of India cultivars trended longer than that of Thailand cultivars. Especially, it was found one rare character in the cultivar Nga Kywe Taung Pyan from Myanmar which possessed an extra long sterile lemma and brown (tawny) lemma and palea. Aroma strength of this cultivar was weaker than others, so it is unnecessary to use it in aromatic rice breeding program.

Variant level and distribution of grain characters differed fairly in this collection. Extra long grains were dominant phenotypes found in Afghanistan, Cambodia, India and Thailand populations. Whereas long grain size was displayed more frequently in Vietnam cultivars, medium grain size was more dominant in Myanmar cultivars. Slender and long grain shape were presented more frequently (78\%) except that Myanmar cultivars which were dominant in medium shape. Short size and bold shape consisted of $5 \%$ and $5 \%$, respectively, were observed in only populations collected from Afghanistan and Myanmar. Populations from Cambodia and India were predominantly light brown pericarp color whereas populations from Myanmar, Thailand and Vietnam were the most white pericarp color. Afghanistan cultivars varied larger with three classes of pericarp color. The absence of many phenotypic classes in pericarp color in this collection reflected either low diversity level between surveyed populations or the result from adaptively selection in domestic zones. Grain characters, especially grain size and shape, are important economic significance characteristics for consumption. However, depend on demands of customers, criterion of trading rice changes largely in market, i.e. satisfactory shape and appearance of grain. Slender and long grain trait are desirable for customers from America, Middle East and Southeast Asia.

In the present study, the trait distribution and phenotypic diversity analysis permit some broad generalization about collection and conservation of aromatic rice cultivars in South and Southeast Asia. However, the distribution of the different traits did not show any apparent trend in general or relative to a particular country. It is possible that there was intervention of conscious human selection for these estimated characters through farmers' distinguish and, domestic and international demand. Also, this could be due to differences in sampling strategy and/or the continuous nature of some traits. The traditional aromatic rice cultivars that command a very high price in domestic and international markets are due to their superior grain characteristics possess a number of undesirable traits such as tall stature, susceptibility to lodging and sensitivity to photoperiod, leading to a low yield potential. Consequently, there have been extensive breeding efforts to improve the yield potential by crossing them with the 
popular improved cultivars. These efforts will lead to the development of new aromatic rice cultivars adapting to local cultivation areas and possessing better properties in yield and quality characteristics. However, there is difficulty in recovering desirable recombinants from the crosses involving aromatic rice cultivars and reversion often to parental types in backcross generations. Therefore, for such efforts to be successful, the genetic variability in segregating populations available to breeding should be assessed as accurately as possible.

\section{Estimation of diversity indices}

Estimates of phenotypic diversity for individual traits, populations and countries are shown in Table 4. The diversity indices H' among populations and countries varied in each of twenty-two traits. Polymorphism was common in varying degrees for most characters, thus implying the existence of a wide range of variation in the materials.

For individual traits, the H' values ranged from 0.00 (monomorphic) for plant height, apiculus color, lemma and palea color, and sterile lemma color to 0.94 (highly polymorphic) for grain size. The highest total diversity index H' pooled over populations by grain size $(0.91)$, grain shape (0.88), culm strength (0.87) and plant height (0.82) whereas the lowest was for ligule color and ligule shape (0.44 and 0.44 , respectively). In general, traits from group of grain characters displayed more variation than other groups by diversity index of total collection.

Unlike the individual trait estimates, the comparison of each group of traits indicated a lower magnitude of differences. The highest variation (1.00) was observed for grain characters in Vietnam cultivars and lowest variation (0.23) for panicle character in Thailand cultivars (Table

Table 4. Diversity index (H') of each phenotypic class for countries and overall collection, and mean diversity and its standard error (SE).

\begin{tabular}{|c|c|c|c|c|c|c|c|}
\hline \multirow{2}{*}{ Trait } & \multicolumn{6}{|c|}{ Country } & \multirow{2}{*}{ Total } \\
\hline & Afghanistan & Cambodia & India & Myanmar & Thailand & Vietnam & \\
\hline Leaf & 0.62 & 0.55 & 0.34 & 0.67 & 0.48 & 0.84 & 0.79 \\
\hline Blade pubescence & 0.66 & 0.44 & 0.32 & 0.51 & 0.50 & 0.90 & 0.71 \\
\hline Basal leaf sheath color & 0.46 & 0.50 & 0.28 & 0.54 & 0.44 & 0.57 & 0.54 \\
\hline Flag leaf angle & 0.37 & 0.40 & 0.28 & 0.54 & 0.28 & 0.77 & 0.70 \\
\hline Ligule & 0.83 & 0.85 & 0.66 & 0.93 & 0.73 & 0.99 & 0.88 \\
\hline Ligule color & 0.59 & 0.56 & 0.50 & 0.59 & 0.50 & 0.62 & 0.44 \\
\hline Ligule shape & 0.51 & 0.56 & 0.50 & 0.59 & 0.50 & 0.62 & 0.44 \\
\hline Collar color & 0.51 & 0.56 & 0.32 & 0.59 & 0.50 & 0.98 & 0.80 \\
\hline Auricle color & 0.60 & 0.67 & 0.61 & 0.70 & 0.61 & 0.84 & 0.62 \\
\hline Culm & 0.63 & 0.48 & 0.46 & 0.57 & 0.28 & 0.89 & 0.96 \\
\hline Plant height & 0.66 & 0.44 & 0.50 & 0.51 & 0.00 & 0.82 & 0.82 \\
\hline Culm angle & 0.43 & 0.37 & 0.41 & 0.50 & 0.41 & 0.55 & 0.70 \\
\hline Culm strength & 0.43 & 0.37 & 0.26 & 0.34 & 0.26 & 0.96 & 0.87 \\
\hline Panicle & 0.41 & 0.38 & 0.26 & 0.52 & 0.23 & 0.86 & 0.88 \\
\hline Panicle type & 0.41 & 0.44 & 0.32 & 0.51 & 0.32 & 0.62 & 0.72 \\
\hline Secondary branching & 0.46 & 0.40 & 0.28 & 0.60 & 0.00 & 0.98 & 0.83 \\
\hline Grain & 0.93 & 0.95 & 0.55 & 0.97 & 0.71 & 1.00 & 1.00 \\
\hline Awning & 0.43 & 0.46 & 0.26 & 0.43 & 0.41 & 0.65 & 0.69 \\
\hline Awn color & 0.39 & 0.42 & 0.11 & 0.39 & 0.36 & 0.47 & 0.64 \\
\hline Apiculus color & 0.19 & 0.42 & 0.00 & 0.50 & 0.23 & 0.50 & 0.67 \\
\hline Lemma and palea color & 0.35 & 0.29 & 0.00 & 0.17 & 0.00 & 0.70 & 0.63 \\
\hline Lemma and palea pubescence & 0.43 & 0.37 & 0.26 & 0.43 & 0.26 & 0.66 & 0.72 \\
\hline Sterile lemma color & 0.46 & 0.40 & 0.00 & 0.60 & 0.00 & 0.77 & 0.72 \\
\hline Sterile lemma length & 0.43 & 0.37 & 0.26 & 0.34 & 0.26 & 0.82 & 0.76 \\
\hline Grain size & 0.87 & 0.40 & 0.28 & 0.37 & 0.58 & 0.94 & 0.91 \\
\hline Grain shape & 0.87 & 0.50 & 0.44 & 0.46 & 0.58 & 0.88 & 0.88 \\
\hline Perdicap color & 0.39 & 0.33 & 0.23 & 0.31 & 0.23 & 0.51 & 0.62 \\
\hline Mean \pm SE & $0.68 \pm 0.09$ & $0.64 \pm 0.11$ & $0.46 \pm 0.07$ & $0.73 \pm 0.09$ & $0.49 \pm 0.10$ & $0.92 \pm 0.03$ & $0.90 \pm 0.04$ \\
\hline
\end{tabular}


4). For total diversity index, all characters showed high diversity and less variation from 0.79 for leaf character to 1.00 for grain character. On the countries basis, the highest mean diversity (0.92) was obtained from Vietnam and the lowest one (0.46) is from India. Agro-ecological zone identified as Vietnam displayed the highest diversity over all characters $\left(H^{\prime}=0.84-1.00\right)$. The lower diversity index for countries was found in India and Thailand populations which were probably associated with the natural and artificial selective pressures in addition to sample size. India and Thailand cultivars (Basmati 370, Jasmine 85 and Khao Dawk Mali 105) which estimated in the present study are well-known aromatic rice cultivars and grown popularly now because they are domesticated through the result of long-term selection with varying desired objectives.

The analysis of variance of diversity ( $\left.\mathrm{H}^{\prime}\right)$ for five groups of morphological traits was performed (Table 5). All variation was due to variations between countries rather than within countries. Leaf and grain traits showed significantly difference $(\mathrm{P}<0.01)$ between countries. Others were different significantly at 0.05 level. The diversity might indicate the presence of important genes for breeding purposes of aromatic rice in future.
This finding reflected that grain traits are considerably important characteristics for conservation and utilization in aromatic rice breeding programs.

\section{Cluster analysis}

The Euclidean dissimilarity coefficients of 36 aromatic rice cultivars ranged between 1.41 and 4.24. Bala Laghman, Bala Doshi and Pashadi Laghman had the highest dissimilarity indices of 4.24 in all tested cultivars. Four Cambodia cultivars, Jasmine 85 and Khao Dawk Mali 105 from Thailand, and many of Vietnam cultivars were the closet cultivars with dissimilarity estimates of 1.41 whereas Daw Dam from Thailand showed largest dissimilarity. Cluster analysis placed the 36 cultivars into five clusters having 1 to 22 cultivars per group (Fig. 1, Table 6).

The first cluster consisted of three cultivars Bala Laghman, Bala Doshi and Pashadi Laghman originating from Afghanistan. These were characterized by very weak stem strength, awning and slender grains and colorless pericarp. The second cluster included two cultivars Luke Andrab and Monda Laghman collected from Afghanistan and five Myanmar cultivars. These cultivars displayed moderate strength of stem, awnless grain, grownish

Table 5. Mean squares from the analysis of variance for diversity index ( $\left.H^{\prime}\right)$ for each group of traits.

\begin{tabular}{lcc}
\hline Group of traits & Between country $(\mathrm{df}=5)$ & Within countries $(\mathrm{df}=30)$ \\
\hline Leaf & $0.069^{* *}$ & 0.011 \\
Ligule & $0.039^{*}$ & 0.010 \\
Culm & $0.102^{*}$ & 0.022 \\
Panicle & $0.098^{*}$ & 0.020 \\
Grain & $0.300^{* *}$ & 0.026 \\
\hline
\end{tabular}

Table 6. Clustering of 36 South and Southeast Asia aromatic rice cultivars based on linkage distance.

\begin{tabular}{|c|c|c|c|}
\hline Cluster & No. of cultivars & Cultivars & Origin \\
\hline $\mathrm{I}$ & 3 & Bala Laghman, Bala Doshi, Pashadi Laghman & Afghanistan \\
\hline \multirow[t]{2}{*}{ II } & 7 & Luke Andrab, Monda Laghman, & Afghanistan \\
\hline & & $\begin{array}{l}\text { Nga Kywe Taung Pyan, Nga Kywe Yin, Mee Done Taung, Paw San Shwe } \\
\text { War, Mee Done Hmwe }\end{array}$ & Myanmar \\
\hline \multirow[t]{3}{*}{ III } & 22 & Phkarum Doul, Phkarum Check, Phkarum Chang, Senpidao, & Cambodia \\
\hline & & Jasmine 85, Khao Dawk Mali 105, & Thailand \\
\hline & & $\begin{array}{l}\text { MTL 250-1, MTL 250-2, Gie Vang 2, Nang Tet 2, Thanh Tra, Hoa Lai, Nang } \\
\text { Thom Duc, Nang Thom Som, Nang Huong 2, Nang Thom Cho Dao 3, Nang } \\
\text { Thom Cho Dao 4, Tau Huong 2, Me Huong 2, Nang Thom Cho Dao 1, Nang } \\
\text { Thom Cho Dao 2, VD20 }\end{array}$ & Vietnam \\
\hline IV & 1 & Daw Dam & Thailand \\
\hline $\mathrm{V}$ & 3 & Basmati 370-1, Basmati 370-2, Surjamkhi & India \\
\hline
\end{tabular}




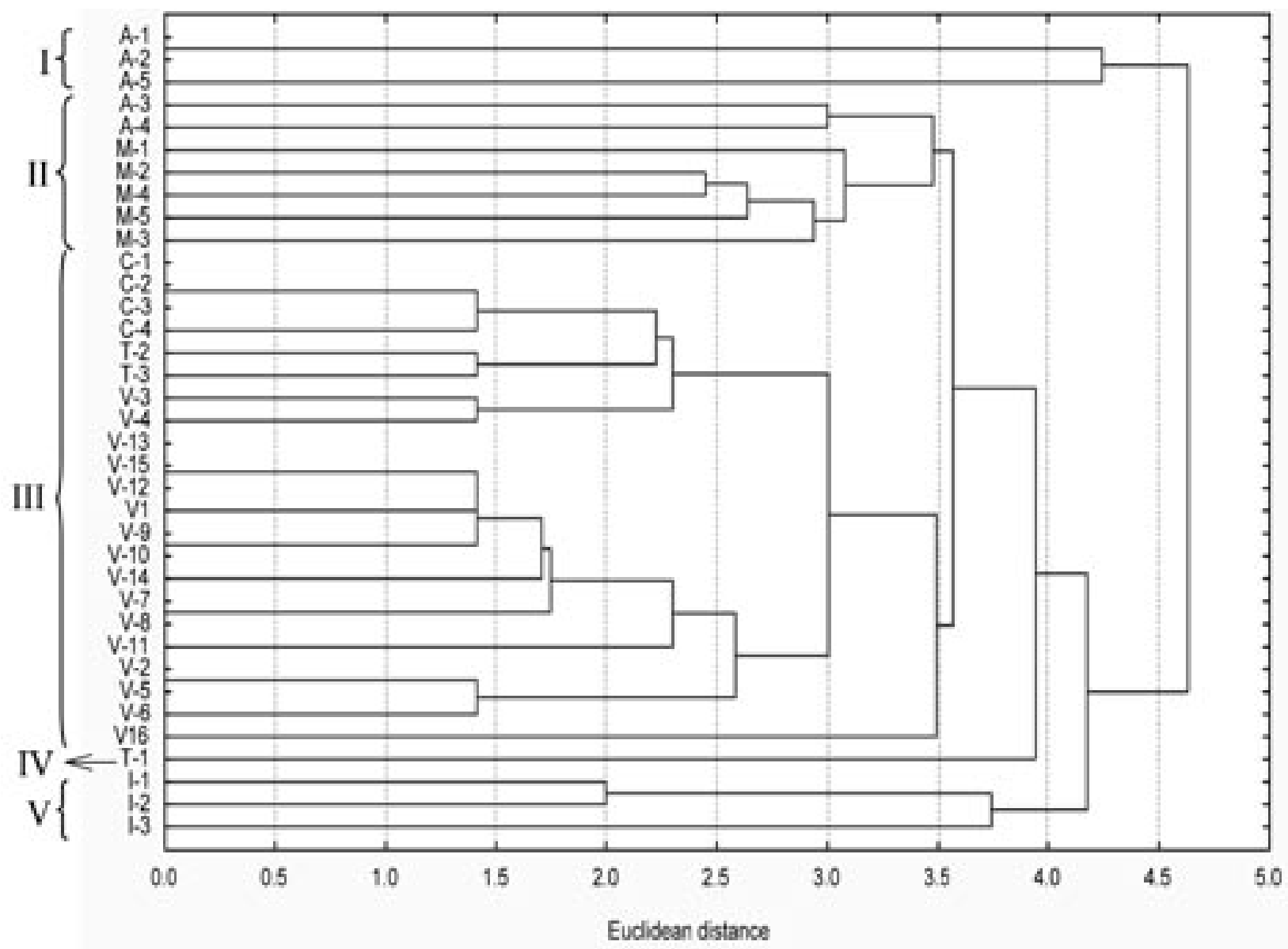

Fig. 1. Dendrogram of 36 Asian aromatic rice cultivars derived by UPGMA from distance matrix of 101 morphometric descriptors from 22 morphological traits. Codes of cultivars are showed in Table 1.

lemma and palea color and medium to bold grain shape. Cluster 3 comprised of four Cambodia, two Thailand and 16 Vietnam cultivars. They were tall plant, awnless to short and partly awning grains, and slender and long grain shape. The fourth cluster consisted only Daw Dam from Thailand. This cultivar was distinct from intermediate plant height, horizontal flag leaf angle, medium grain shape and especially very different color of grain characters. The fifth cluster included three cultivars originated from India. They exhibited sensitive to lodging, open panicle type, light secondary branching in panicle, long sterile lemma length and extra long grain size.

Although cluster analysis grouped cultivars together with greater morphological similarity, the clusters did not necessarily include all the cultivars from the same origin. It was reported that no association between morphological characters and geographical origin (Dias et al. 1993; Amurrio et al. 1995). Revilla and Tracy (1995) also observed a low level of morphological variability among widely used open-pollinated sweet corn cultivars.
It is possible that the exchange of germplasm between the neighboring regions has been practiced and perhaps some cultivars originated from the same ancestor.

\section{CONCLUSIONS}

Up to now, phenotypic diversity in Asian aromatic rice cultivars has not been studied widely. The present study indicated that the amounts of variability were distributed in agro-ecological zones and highlighted the strong difference among populations. Cultivar collection of this study as indicative source of diversity should take account of distribution of polymorphism. Priorities of germplasm collecting should focus on countries with relatively large variation, i.e. Vietnam, Myanmar, Afghanistan, with due consideration to the cause of genetic erosion and environmental degradations in Asia countries.

Morphological variation does not always reflect real genetic variation because of interaction between genotype and environment, and the large unknown genetic control of polygenic morphological and agronomic traits (Smith 
and Smith, 1992). Beyene et al. (2005) also identified that the morphological traits were relatively less efficient for precise discrimination of closely related accessions and analysis of their genetic relationships. Despite this limitation, morphological traits are useful for preliminary evaluation because it is fast, simple, and can be used as a general approach for assessing genetic diversity among morphologically distinguishable cultivars.

Because morphological variation alone does not reflect the total variation which is necessary for breeding new aromatic rice cultivars from demands of production and customers, further comprehensive investigation including markers such as isozyme, protein, molecular markers and quantitative characters will probably provide a complete view about the genetic variation of aromatic rice cultivars. In addition, amount of cultivars with larger agro-ecological distribution must be increased in order to estimate the association between different geographic distance and genetic distance to morphologic variables and others.

In addition favorite 'aroma' flavor, the aromatic rice cultivars have to be improved other quality characteristics such as grain appearance, cooking and eating quality, and nutrient content in meeting with the demands of consumers for healthy high-quality food. Therefore, it is necessary to address the correlation between quality characteristics and phenotypic variations before aromatic breeding program is undertaken.

ACKNOWLEDGEMENTS We thank College of Agriculture and Applied Biology (Can Tho University, Vietnam) and MAFF Gene Bank (Japan) for supplying seeds. We also thank Dr. Takashi MOTOBAYASHI (Honmachi Farm, TUAT, Japan) for field cultivation.

\section{REFERENCES}

Amurrio, J.M., de Ron, A.A. \& Zeven, A.C. 1995. Numerical taxonomy of Iberian pea landraces based on quantitative and qualitative characters. Euphitica, 82: 195-205.

Beyene Y., Boths, A.M. \& Myburg, A.A. 2005. A comparative study of molecular and morphological methods of describing genetic relationships in traditional Ethiopian highland maize. African Journal of Biotechnology, 4: 586-595.

Dias, J.S., Monteiro, A.A. \& Lima, M.B. 1993. Numerical taxonomy of Portuguese Tronchuda cabbage and Galega kale landraces using morphological characters. Euphitica, 69: 51-68.
Hien, N.L. 2001. Effect of direct sowing densities on the characteristics of the culm related to lodging resistance in Vietnam paddy rice. Master Thesis, Tokyo University of Agriculture and Technology (Tokyo, Japan) 58p.

Hutchenson, K. 1970. A test for comparing diversities based on the Shannon formular. Journal of Theorical Biology, 29: 151-154.

IRRI-IBPGR. 1980. Descriptors for rice (Oryza sativa L.). IRRI, P. O. Box 933, Manila, Philippines. 21pp.

Kurlovich, B.S. 1998. Species and intraspecific diversity of white, blue and yellow lupins. Plant genetic Resource Newsletters, 115: 23-32.

Ookawa, T. \& Ishihara, K. 1993. Varietal differences in physical characteristics of the culm related to lodging resistance in paddy rice. Japanese Journal of Crop Science, 61: 419-425.

Perry, M.C. \& McIntosh, M.S. 1991. Geographical patterns of variation in the USDA soybean germplasm collections. I. Morphological traits. Crop Science, 31: 1350-1355.

Revilla, P. \& Tracy, W.F. 1995. Morphological characterization and classification of openpollinated sweet corn cultivars. The Journal of The American Society For Horticultural Science, 120: 112-118.

Ruiz, M. Carrllo, J.M. \& Varela, F. 1997. Relationship between some geographical parameters and agro/ morphological and biochemical characters in a sample of Spanish landraces of barley (Hordeum vulgare L.). Plant Genetic Resource Newsletter, 112: 86-89.

Smith, J.S.C. \& Smith, O.S. 1989. The description and assessment of distances between inbred lines of maize: The utility of morphological, biochemical and genetic descriptors and a scheme for the testing of distinctiveness between inbred lines. Maydica, 34: 151-161.

Smith, J.S.C. \& Smith, O.S. 1992. Fingerprinting crop varieties. Advance Agronomy, 47: 85-140.

Smith, S.E., Al Doss, A. \& Warburton, M. 1991. Morphological and agronomic variation in North African and Arbian alfalfas. Crop Science, 31: 1159-1163.

Sood B.C. \& Siddiq, E.A. 1978. A rapid technique for scent determination in rice. Indian Journal of Genetic and Plant Breeding, 38: 268-271.

Takahashi, N., Alterfa, H.A.H. \& Sato, T. 1986. Significant role of awn in rice plants (1). A survey for agricultural value of rice awn. Rep. Institute of 
Agricultural Resource Tohoku University, 35: 21-31.

Watanabe, T. 1997. Lodging resistance. Science of the rice plant. Vol.3, Part II, Chap. 4. Food and Agriculture Policy Resource Center. Tokyo, 567-577.

Yoshida, S. 1981. Fundamentals of rice crop science. IRRI, Los Banos, Phillipines.

Received $24^{\text {th }}$ Apr. 2006

Accepted $24^{\text {th }}$ Feb. 2007 